\title{
"Algunas Cuestiones Sobre el Practicum en el Grado de Relaciones LabOrales y Recursos Humanos"
}

\author{
Carmen Ferradans Caramés* \\ José María Pérez Monguió***
}

RESUMEN:

El objetivo de este trabajo es analizar la funcionalidad del practicum, asignatura del grado de Relaciones Laborales y Recursos Humanos, para adquirir determinadas competencias genéricas y específicas. Se describe la idoneidad de las prácticas externas insertas en el plan de estudios del título para aprender algunas competencias que difícilmente se pueden enseñar en el aula.

Para facilitar el cumplimiento de los objetivos previstos con esta asignatura se propone un protocolo de actuación, la existencia de un coordinador de prácticas de la titulación, de un responsable académico de la asignatura y la designación de tutores internos y externos (de empresa) de los estudiantes en prácticas. Asimismo, se proponen unos materiales que ayuden a su evaluación, la memoria de las prácticas realizada por el alumno/a y los cuestionarios de evaluación cumplimentados por el tutor interno y el externo.

Palabras Clave

Practicum; Relaciones Laborales y Recursos Humanos; tutores externos.

* Profesora Titular de Universidad. Departamento Derecho del Trabajo y de la Seguridad Social. Universidad de Cádiz • carmen.ferradans@uca.es

* Prof. Titular de Universidad. Departamento Dcho. Público. Universidad de Cádiz

- josemaria.monguio@uca.es 


\section{ABSTRACT:}

The point of this work is to analyse the functionality of practicum, a subject in the Labor Relations \& Human Resources Degree Course, to acquire some general skills and specific competencies. We describe the appropriateness of work experience as part of the degree course as they allow the student to acquire skills that cannot be taught the same in the classroom.

To make it easy to achieve the set goals with this work experience, we propose Guidelines, the figure of a Work Experience Coordinator for the degree and naming internal tutors and mentors in the company for the work experience students.

We also present some materials that will help in the assessment process, the Report of the Work Experience carried out by the student and evaluation questionnaires to be completed by the external tutor.

KEYWORDS:

Work Experience; Labour Relations; Human Resources; External tutors.

\section{1- INTRODUCCIÓN}

La implantación del Espacio Europeo de Educación Superior pretende dar un giro a los sistemas de enseñanza-aprendizaje universitarios, realzándose la importancia de la formación basada en competencias. La competencia se define como la capacidad de una persona para ejecutar un trabajo cumpliendo con un determinado estándar en un entorno laboral; entendiéndose por capacidad el conjunto de conocimientos, habilidades y actitudes, valores y ética profesionales requeridos para demostrar competencia ${ }^{1}$.

En este sentido, numerosos estudios e informes han propuesto perfiles profesionales y listas de competencias profesionales que deben poseer los egresados de las distintas titulaciones, entre ellos los del grado de Relaciones Laborales y Recursos Humanos. Sobre estas cuestiones existe un consenso amplio en la comunidad universitaria, si bien hay un aspecto relacionado con la formación basada en competencias que consideramos que no está del todo resuelto: su evaluación.

1. Cano García, E.M., "La evaluación por competencias en la educación superior", Profesorado, revista de curriculum y formación del profesorado, vol. 12, n³, 2008, pág. 3. Disponible en http://www.ugr. es/ recfpro/rev123COL1.pdf 
En el entorno académico, en el aula, no se pueden recrear determinado tipo de situaciones (jerarquía, equipos multidisciplinares de trabajo...) y, por lo tanto, el desarrollo de dichas competencias y su evaluación resulta compleja. Asimismo, el profesor universitario en ocasiones no tendrá la formación suficiente para evaluar este tipo de competencias, siendo el reto de formar a futuros profesionales cada vez más complicado.

Es por ello que el practicum, en aquellos títulos que lo poseen, puede aportar un entorno apropiado para el desarrollo y evaluación de estas competencias transversales, fundamentalmente porque el estudiante puede desarrollarlas en un entorno similar a aquél en el que después deberá demostrar que las posee.

Es por ello que es necesario analizar la funcionalidad del practicum como entorno ideal para la puesta en práctica y evaluación de algunas de las competencias requeridas al estudiante del grado de Relaciones Laborales y Recursos Humanos, así como plantear algunas propuestas que contribuyan al desarrollo eficaz del mismo, fundamentalmente en lo que se refiere al papel de los tutores internos (profesores), los tutores externos (tutores en la empresa) y los alumnos.

\section{2.- LA FORMACIÓN EN COMPETENCIAS}

Actualmente vivimos en unos tiempo de avance continuo, cambios constantes y competitividad en la mayoría de las facetas de la vida.

Uno de los objetivos de la Universidad es dotar a la sociedad del capital humano que deberá introducirse en las empresas y/o desarrollar sus ideas emprendedoras a través de las opciones que ofrece el autoempleo. Por ello, la Universidad debe articular los medios necesarios para que la inserción laboral de los egresados se produzca en las mejores condiciones posibles y, además, comprobar que éstos responden a las demandas de las empresas de nuestro tejido productivo y que tienen la capacidad de adaptación continua a los cambios.

El conjunto de capacidades, habilidades y actitudes complementarias a la formación técnica-teórica forma parte de lo que se viene denominando como competencias profesionales ${ }^{2}$, que no sólo consideran al componente de conocimiento profesional (saber), sino que va más allá incluyendo habilidades profesionales (saber hacer), valores, ética y actitudes profesionales (saber ser o estar).

Por lo tanto, adquirir competencias implica la búsqueda de aprendizajes más globales y prácticos, donde los nuevos conocimientos estén dirigidos al desarrollo de la capacidad de actuación de los sujetos, permitiéndoles llevar a cabo procesos de acción cada vez más complejos.

2. La Ley Orgánica 5/2002, de 19 de junio, de las Cualificaciones y de la Formación Profesional entiende por competencia profesional, el conjunto de conocimientos y capacidades que permiten el ejercicio de la actividad profesional conforme a las exigencias de la producción y el empleo. 
La posesión de competencias profesionales se está convirtiendo en un factor determinante de los procesos de selección de las empresas. Es más, las ofertas de empleo cada vez más exigen profesionales con competencias que encajen en labores diferentes, y que permitan que la vida laboral del trabajador vaya cambiando según las variaciones que se producen en el mercado laboral, convirtiéndose la polivalencia, la formación continua y el reciclaje profesional en valores enriquecedores de la población activa.

En este sentido el diseño de los nuevos grados implantados en el sistema universitario adaptado al Espacio Europeo de Educación Superior (EEES) considera necesario que el estudiante adquiera tanto las competencias específicas (cognitivas, profesionales y actitudinales) de su titulación, es decir, en función del perfil académico profesional que reclama la sociedad en la que se va a insertar; como las genéricas o transversales (instrumentales, personales y sistémicas), comunes a la mayoría de las profesiones y que se relacionan con la puesta en práctica integrada de aptitudes, conocimientos y valores adquiridos.

La adquisición del conjunto de competencias señaladas, que procuran la mejor preparación del alumno/a para el ejercicio de actividades de carácter profesional, será más óptima si en el programa formativo coexiste un período de educación formal con uno de experiencia práctica. Este período de experiencia práctica, previo a la inserción laboral del estudiante, practicum, permitirá a éste profundizar en la adquisición de conocimientos, habilidades profesionales y actitudes, así como demostrar la adquisición de dichas competencias, es decir, su competencia 3 .

La evaluación de las competencias es una tarea muy importante del proceso formativo, si bien hasta el momento no hay disponibles muchos materiales para esta tarea. Cada titulación tiene claramente definidas sus competencias, resultando en principio adecuadas y claras, pero son insuficientes los materiales existentes para evaluar competencias. Por ello, se hace necesaria la articulación de dispositivos válidos y fiables que contribuyan a evidenciar que el alumno/a posee las competencias profesionales requeridas, conocidos por todos profesores, alumnos y en el caso del practicum también por los tutores de empresa.

Para el practicum, de forma específica, se distinguen al menos tres tipos de evidencias de evaluación ${ }^{4}$ :

- Evidencias sobre el conocimiento, que pueden ser evaluables a través de pruebas teórico-prácticas. Aquí debe jugar un papel fundamental el tutor

3. La competencia implica demostrar que se posee la habilidad de desempeñar una tarea en el marco estándar de actuación requerido y debe ser constatable en la práctica, mediante comportamientos evidenciables.

4. Tejada Fernández, J., "El trabajo por competencias en el practicum: cómo organizarlo y cómo evaluarlo", pág. 22. VIII Symposium Internacional sobre Practicum y Prácticas en empresas en la formación universitaria, Poio, 30 junio-2 julio 2005. Disponible en http://webs2002.uab.es/paplicada/ $\mathrm{htm} /$ papers/Trabcompetenciaspracticum.pdf 
académico, ya que la experiencia práctica debe ser evaluada por el mismo en base a la documentación escrita revisada por él y a las exposiciones orales de los alumnos/as en prácticas.

- Evidencias sobre el proceso, que se corresponden con los elementos que indican la calidad de ejecución de una tarea. Para este tipo de evidencias es esencial el rol desempeñado por el tutor de empresa, que deberá observar y analizar la destreza del alumno/a dentro del proceso de trabajo.

- Evidencias del producto, que corresponden a los resultados o productos identificables y tangibles, y que pueden usarse como referentes para demostrar que una actividad fue realizada.

A estos efectos, en el siguiente apartado se va a describir de forma más concreta algunas de las características que rodean al practicum del Grado de Relaciones Laborales y Recursos Humanos, procurando proponer herramientas que orienten la adquisición de determinadas competencias en el marco del mismo.

\section{3.- EL PRACTICUM Y SU EVALUACIÓN EN EL GRADO DE RELACIONES LABO- RALES Y RECURSOS HUMANOS}

\section{1.- Perfil profesional del graduado}

Este título proporciona una habilitación profesional que se corresponde con numerosas profesiones y funciones dentro de las organizaciones productivas. Esto es así, porque tradicionalmente una de las características más emblemáticas de estos estudios es el carácter multidisciplinar de sus contenidos formativos, que recogen distintas perspectivas y análisis sobre la realidad laboral desde las áreas jurídicas, económicas, de organización de empresas, de psicología, de sociología, de historia, etc.

El Libro Blanco de la titulación ${ }^{5}$ analiza y valora, a efector de delimitar el perfil profesional, los siguientes elementos y fuentes:

- El perfil curricular y profesional de los actuales Diplomados en Relaciones Laborales y Licenciados en Ciencias del Trabajo.

- La información sobre perfiles profesionales derivados de titulaciones afines en el contexto europeo.

- La documentación elaborada al respecto por el Pleno de Decanos y Directores de Centros Universitarios responsables de estas titulaciones.

5. Libro Blanco del Título de Grado en Ciencias Laborales y Recursos Humanos, disponible en http:// www.aneca.es/var/media/150308/libroblanco_rrhh_def.pdf 
La valoración realizada de estos perfiles por los egresados en la encuesta de inserción laboral.

Asimismo, según lo dispuesto en el RD 1429/1990, los objetivos formativos del título de Diplomado en Relaciones Laborales "deberán proporcionar una formación adecuada en las bases teóricas y en las técnicas de la organización del trabajo y de la gestión del personal; así como de la ordenación jurídica del trabajo y de la Seguridad Social'. Dos grandes áreas o sectores de actividad se desprenden del objetivo formativo de esta titulación:

1. La organización funcional del trabajo y la gestión de los recursos humanos en la empresa, dentro de una organización empresarial, o como consultor externo especializado.

2. La actuación en materia jurídico laboral, posibilitando el asesoramiento laboral en el ejercicio de la profesión de Graduado Social.

En el estudio realizado en el Libro Blanco sobre titulaciones afines en el contexto europeo, también se han analizado las distintas salidas y capacitaciones profesionales que presentan estas titulaciones homólogas, que se pueden sintetizar en: Directores o técnicos superiores de recursos humanos en los staff directivos de las empresas, responsables directos de las políticas de gestión y administración de los recursos humanos; Profesionales liberales expertos en el asesoramiento jurídico-laboral, en la organización del trabajo y de la producción, así como en la representación de intereses colectivos y profesionales, tanto en el ámbito privado como en la administración pública; Expertos en el campo de las políticas laborales promovidas por la administración pública como responsables de políticas sociolaborales locales, regionales, nacionales; Consultores y/o auditores sociolaborales; Profesionales especializados en afrontar los desafíos y oportunidades que implican los procesos de reestructuración productivos, los cambios en la estructura de los mercados, la integración europea y las aportaciones de los nuevos enfoques en el mundo del trabajo y de la organización del empleo; Profesionales con capacidad de insertarse en equipos multidisciplinares, en el ámbito del asesoramiento, de la consultoría o de las empresas; Enseñanza secundaria y superior, universitaria y no universitaria.

Asimismo, se tomó en consideración la documentación elaborada por el Pleno de Decanos y Directores responsables de estas titulaciones. En dicha documentación se postulan tres bloques o campos de actuación profesional preferentes y también se determinan otros campos de actuación profesional u orientaciones ocupacionales vinculadas a estos estudios: Asesoramiento jurídico-laboral; Dirección y Gestión de Recursos Humanos; Intervención en el mercado de trabajo y desarrollo de Políticas Socio-Laborales; Nuevas áreas especializadas que demanda el mercado de trabajo cuya capacitación profesional se solicita a la formación universitaria: expertos en salud laboral y prevención de riesgos laborales, expertos en auditoría sociolaboral, expertos en políticas de protección social y de seguridad social, expertos en el conocimiento de los sistemas de relaciones laborales de nuestro entorno para el asesoramiento de nuestras empresas. 
Del conjunto de datos analizados, a efectos del título de grado en Relaciones Laborales y Recursos Humanos, se concretan siete perfiles profesionales o ámbitos de actuación profesional para los nuevos titulados:

1. Graduado Social.

2. Dirección y Gestión de Recursos Humanos.

3. Gestión, mediación e intervención en el mercado de trabajo: agentes de empleo y desarrollo local.

4. Prevención de Riesgos Laborales.

5. Administraciones Públicas.

6. Auditoría sociolaboral.

7. Enseñanza.

Por lo tanto, en el diseño del grado se procura ofrecer al egresado una base conceptual y unas competencias y habilidades que puedan ser desplegadas en múltiples espacios profesionales, tales como los recursos humanos, el diseño y la gestión de políticas (públicas y privadas) de formación, empleo, inserción laboral, la orientación profesional, la intermediación en el mercado de trabajo, la consultoría y asesoría laborales y la dirección y gestión de relaciones laborales.

\section{2.- LA ADQUISICIÓN DE COMPETENCIAS EN EL PRACTICUM}

Para la inserción laboral del graduado en Relaciones Laborales y Recursos Humanos en uno de los perfiles descritos, debe poseer, entre otras, las competencias básicas de organización y planificación, análisis y síntesis, toma de decisiones, trabajo en equipos de carácter multidisciplinar y de resolución de problemas, que le capacitan para afrontar con éxito su incorporación al mercado laboral ${ }^{6}$.

Igualmente debe adquirir las competencias específicas, cognitivas (saber), profesionales procedimentales (saber hacer) y académicas actitudinales (ser).

Resulta interesante, a estos efectos, un proyecto realizado por la Dirección General de Empleo y el Consejo Social de la Universidad de Cádiz’ , que pretende identificar los valores y competencias demandadas en el mercado profesional del entorno socioeconómico de la Universidad de Cádiz y diseñar e implementar un programa de formación competencial, de carácter transversal, acorde a las necesidades detectadas en el entorno laboral, para consolidar la formación en competencias para el empleo

6. Vid, a modo de ejemplo, el cuadro de competencias generales (instrumentales, personales y sistémicas), expuesto en la memoria del grado de Relaciones Laborales y Recursos Humanos de la Universidad de Cádiz, disponible en http://www.uca.es/centro/1C16/centro/1C16/documentos/Memoria\%20Grado.

7. Proyecto de Implantación de un Programa de Formación y Certificación de Competencias para titulados universitarios, Dirección General de Empleo y Consejo Económico y Social UCA, 2010. 
como una parte crucial del itinerario de los estudiantes de la UCA. El proyecto ha tenido varias fases, habiéndose dedicado una de ellas al establecimiento de los perfiles básicos de competencias por áreas de conocimiento.

En los resultados del área jurídica se resalta la importancia de competencias tales como la adaptabilidad al cambio, el aprendizaje continuo, el compromiso con la empresa, la orientación al cliente, la asertividad, la capacidad de comunicación, la organización y planificación, la calidad, el desarrollo de personas y la iniciativa. Y en el área social se destacan competencias tales como la capacidad de comunicación, la capacidad de gestión, la iniciativa, la responsabilidad, la adaptabilidad al cambio, la asertividad, la creatividad, la credibilidad técnica, la organización y planificación y la resolución de problemas.

Algunas de las competencias indicadas difícilmente pueden ser adquiridas y, en su caso, evaluadas en las aulas, pudiendo resultar, sin embargo, el desarrollo del practicum previsto en la titulación un instrumento válido para ello.

La memoria del grado de Relaciones Laborales y Recursos Humanos de la Universidad de Cádiz describe en el módulo XIII la asignatura Prácticas, que consta de seis créditos ECTS. Su desarrollo se produce en el segundo semestre del cuarto curso y tiene carácter obligatorio. Como requisito previo a la matriculación del alumno/a en esta asignatura se le exige haber superado sesenta créditos de la formación básica y 120 de la formación obligatoria, lo que indica que el estudiante debe tener un amplio número de créditos aprobados y que, por ende, en el momento en que inicia sus prácticas en una empresa o Administración ya tiene unos conocimientos teóricoprácticos extensos de las distintas asignaturas de la titulación.

Las competencias que se pretende que el alumno adquiera con este módulo son las siguientes:

\section{Competencias Generales}

\begin{tabular}{|c|c|}
\hline Competencia 1 & Capacidad de análisis y síntesis \\
\hline Competencia 2 & Capacidad de organización y planificación \\
\hline Competencia 3 & Habilidad de comunicación oral y escrita en lengua nativa \\
\hline Competencia 4 & Conocimientos de informática relativos al ámbito de estudio \\
\hline Competencia 5 & Capacidad para gestionar la información \\
\hline Competencia 6 & Capacidad para la resolución de problemas \\
\hline Competencia 7 & Destreza para el trabajo en equipos \\
\hline Competencia 8 & Capacidad para trabajar en un equipo de carácter interdisciplinar \\
\hline Competencia 9 & Capacidad de trabajo en un contexto internacional \\
\hline Competencia 10 & Habilidades en las relaciones interpersonales \\
\hline Competencia 11 & Capacidad de razonamiento crítico y autocrítico \\
\hline Competencia 12 & Capacidad de aprendizaje y trabajo autónomo \\
\hline Competencia 13 & Capacidad para aplicar los conocimientos a la práctica \\
\hline Competencia 14 & Cadecisiones \\
\hline
\end{tabular}


Competencias Específicas

\begin{tabular}{|c|c|}
\hline Competencia 1 & $\begin{array}{c}\text { Capacidad para conocer las principales fuentes estadísticas en materia } \\
\text { sociolaboral }\end{array}$ \\
\hline Competencia 2 & $\begin{array}{c}\text { Capacidad de transmitir y comunicarse por escrito y oralmente usando la } \\
\text { terminología y las técnicas adecuadas }\end{array}$ \\
\hline Competencia 3 & $\begin{array}{c}\text { Capacidad de aplicar las tecnologías de la información y la comunicación en } \\
\text { diferentes ámbitos de actuación }\end{array}$ \\
\hline Competencia 4 & $\begin{array}{c}\text { Capacidad para seleccionar y gestionar información y documentación } \\
\text { laboral }\end{array}$ \\
\hline Competencia 5 & $\begin{array}{l}\text { Capacidad para interrelacionar las distintas disciplinas que configuran las } \\
\text { relaciones laborales }\end{array}$ \\
\hline Competencia 6 & $\begin{array}{l}\text { Capacidad para interpretar datos e indicadores socioeconómicos relativos al } \\
\text { mercado laboral }\end{array}$ \\
\hline Competencia 7 & $\begin{array}{l}\text { Capacidad para comprender el carácter dinámico y cambiante de las } \\
\text { relaciones laborales en el ámbito nacional e internacional }\end{array}$ \\
\hline Competencia 8 & Habilidad para aplicar los conocimientos a la práctica \\
\hline Competencia 9 & $\begin{array}{c}\text { Capacidad para comprender la relación entre procesos sociales y la } \\
\text { dinámica de las relaciones laborales }\end{array}$ \\
\hline
\end{tabular}

Las prácticas deber permitir a los estudiantes, entre otros resultados, descubrir, analizar y comprender el contexto y los procesos de asesoramiento jurídico-laboral, la dirección y gestión de recursos humanos, la intervención en el mercado de trabajo y el desarrollo de políticas socio-laborales, la gestión en salud laboral y prevención de riesgos laborales, la auditoría de recursos humanos, las políticas de protección social y de seguridad social, etc.

\section{3.- HerRamientas y materiales PARA SU EVALUACión}

Para guiar el aprendizaje del alumno/a en el practicum hay que instaurar un procedimiento. El grado de Relaciones Laborales y Recursos Humanos debe tener un responsable, coordinador, de prácticas de la titulación, y la asignatura Prácticas del mismo un responsable académico. Al mismo tiempo será necesario designar un tutor interno o tutor académico para cada alumno/a, uno de los profesores del Grado, que se encargará de la orientación y supervisión del estudiante asignado, así como de ayudarlo en la adquisición de las competencias. Asimismo, también se deberá nombrar un tutor de empresa, cuya labor será dirigir al alumno hacia la consecución de los objetivos formativos previamente establecidos y ayudarle en la adquisición y desarrollo de las competencias profesionales.

El modelo descrito es el que ha funcionado en la Universidad de Cádiz en las prácticas extracurriculares y parece acertado implantarlo, con algunas propuestas de mejora, en las prácticas curriculares. 
La primera tutoría entre el tutor interno y el alumno debería destinarse a explicar al alumno qué actividades debe desarrollar en las prácticas, así como que a su finalización deber realizar un informe sobre las mismas, asesorándole sobre este último extremo; sería conveniente enseñar al alumno/a los cuestionarios de evaluación que deberán elaborar al final de la práctica tanto el tutor externo como el interno, que servirán de guía al estudiante sobre cuáles son las competencias que se le van a evaluar en la práctica y que, por lo tanto, deberá adquirir y demostrar en el desempeño. En la segunda tutoría, una vez iniciadas las prácticas en la empresa o Administración adjudicada, el alumno deberá describir al tutor el plan formativo que se está desarrollando y durante ésta y, en su caso, otra sucesiva el tutor interno realizará un seguimiento de la evolución del alumno en la práctica (la duración media de la práctica será de entre dos y tres meses).

En la última tutoría el estudiante entregará al tutor interno un borrador de su informe final, para que el tutor pueda revisarla y plantearle sugerencias de mejora para su entrega final.

El tutor de empresa procurará supervisar el aprendizaje del alumno, día a día, en la práctica. Al concluir la misma deberá cumplimentar un cuestionario en el que evalúe tanto la capacidad del alumno/a para aplicar los conocimientos adquiridos como determinadas competencias profesionales. El cuestionario servirá de guía al tutor, para conocer qué destrezas y/o habilidades debe observar y evaluar del estudiante en prácticas.

A estos efectos resulta fundamental la comunicación previa entre tutor interno y externo, para que aclaren cuál es el plan formativo que se va a seguir y cuáles son las competencias profesionales a evaluar. Asimismo, esta información debe ser transmitida al alumno, para que conozca qué contenidos abarca su evaluación.

Así pues, los materiales esenciales para la evaluación de las prácticas por el responsable académico de la asignatura son: la memoria realizada por el alumno/a, el cuestionario de evaluación del tutor interno, que se basa en las reuniones de seguimiento realizadas, y el cuestionario de evaluación del tutor externo, basado en la observación del trabajo diario del estudiante.

\section{4.- $\quad$ CONCLUSIONES}

El graduado en Relacionas Laborales y Recursos Humanos tiene que formarse en determinadas competencias profesionales que facilitarán su inserción laboral. El listado de competencias profesionales, genéricas y específicas, que debe adquirir el estudiante es detallado de forma bastante consensuada en el Libro Blanco de la titulación y en la memoria del grado adoptada en cada Universidad en la que se va a impartir. En esta comunicación se analiza cómo la adquisición de algunas de las com- 
petencias indicadas puede ser facilitada por la realización de la asignatura Prácticas, es decir mediante el desarrollo de prácticas externas en empresas o Administraciones con distintos perfiles profesionales, asesoramiento legal de empresas, prevención de riesgos laborales, gestión de recursos humanos...

Independientemente del lugar de desarrollo de las prácticas se debe instaurar un procedimiento similar para todos los alumnos, que garantice que la formación recibida en esta asignatura sea adecuada.

Por ello, se propone que junto al responsable académico de la asignatura se proceda a la designación de tutores internos y externos (de empresa) y que existan unos determinados documentos que ayuden a la evaluación de las prácticas, la memoria o informe elaborado por el estudiante, el cuestionario de evaluación del tutor interno y el del externo, para que en su conjunto permitan valorar si se han obtenido los objetivos pretendidos con el practicum.

Asimismo, creemos que es fundamental que exista una comunicación fluida entre el tutor interno y el externo, para poner en común las competencias a evaluar e informar y trasmitir al alumnado de forma clara qué destrezas y habilidades aplicados en la práctica se le van a evaluar.

Todo esto puede requerir la realización de unas sesiones formativas para los profesores implicados en el practicum, donde se les exponga la metodología a emplear y las herramientas que se van a utilizar, para que los alumnos obtengan el mayor provecho posible del desarrollo de las competencias inherentes al practicumy conozcan de forma transparente la forma de su evaluación. 